\title{
User inequity implications of road network vulnerability
}

\author{
Erik Jenelius \\ Royal Institute of Technology, Sweden ${ }^{a}$
}

\begin{abstract}
An important purpose of the road transport system is to allow people to commute in efficient and reliable ways. For various undesired reasons, however, link capacities are sometimes reduced or links are closed completely. To assess and reduce the risk of such events, a key issue is to identify road links that are particularly important, i.e. roads where disruptions would have particularly severe consequences. This paper presents a method for incorporating user equity considerations into a road link importance measure. As a complement to measuring the total increase in vehicle travel time, we also measure the disparity in the distribution among individual users. These two components are combined to form an equity-weighted importance measure. We study the properties of this measure both analytically and in a full-scale case study of the Swedish road network. A main result is that increasing the weight put on the equity aspect transfers importance from the main roads to smaller local roads. The use of the measure in transport policy and planning is discussed.
\end{abstract}

Keywords: Transport; Networks; Efficiency; Equity; Vulnerability; Reliability

\section{Introduction}

The road transport system has to fulfill a number of different purposes, one of the most important being to allow people to commute efficiently and reliably. The road network may be able to meet this demand under ideal conditions, i.e. when all road links are operating at their full capacities. For various reasons, however, link capacities are sometimes reduced or links are closed completely. This may be caused by adverse weather conditions such as heavy precipitation, or by physical breakdowns of the roads due to landslides, flash floods, or general wear and tear, among other causes. Network degradation may also be caused by car crashes or nearby industrial accidents, or even by malevolent attacks. Events such as these may put the road transport system under such strain that the demands from every user cannot be met. Road network vulnerability analysis aims to to assess the probabilities, as well as the economic and societal consequences, of such events.

The main objective of national transport authorities is typically to ensure an economically efficient transport system. For this reason, it is highly desirable to reduce the risk of network degradations as much as possible. A natural first step in this process is to identify road segments that are particularly important-i.e., roads where disruptions would lead to particularly severe consequences. However, transport authorities are often also required to consider distributional aspects in their planning processes. In connection to vulnerability, this suggests that not only

\footnotetext{
ajenelius@infra.kth.se
} 
the overall impacts of network degradation should be considered, but also the distribution of the impacts among the users; in other words, no individuals should be affected too severely, and the inequity between individuals should not be too large. In essence, a road link that, when disrupted, produces a highly inequitable distribution of impacts may well be considered more important than one that produces slightly greater but more evenly distributed impacts when disrupted.

This paper presents a method for quantifying the importance of road links based not only on the overall consequences but also on the disparities among users. The focus is on closures lasting a few days at most, and on the road network outside the most congested urban areas. Our importance measures are based on the increases in user travel times during the closure. Efficiency importance is measured as the sum, and equity importance as the coefficient of variation, of these travel time increases.

The paper is organized as follows. The scope and limitations of the method are stated more precisely in Section 2. We then define measures of link efficiency and equity importance, and an equity-weighted importance measure incorporating both components is proposed. A model for the increases in travel time during a closure is also presented. Some notable properties of the importance measures are shown analytically in Section 3. Section 4 presents a full-scale case study of the Swedish road network, and results from the study are given in Section 5. We conclude in Section 6 by discussing the use of these measures in policy decision-making.

\subsection{Literature review}

Issues of transport reliability and road network vulnerability are now receiving increased attention; see, for example, the special journal issues edited by Lam (1999) and Sumalee and Karauchi (2006), and the books edited by Bell and Cassir (2000), Iida and Bell (2003), and Murray and Greubesic (2007). A review of the field and a framework for vulnerability analysis is given by Berdica (2002). In particular, a number of papers present methods to identify important (critical, significant, vital) links in a road network. Taylor et al. (2006) use three different measures of diminished accessibility: the increase in generalized travel cost, the relative decrease in the Hansen integral accessibility index, and the increase of a "remoteness" index specially developed for the regional and remote parts of Australia. Jenelius et al. (2006) use the increase in generalized travel cost to define various measures of link importance, which are then applied to the road network of northern Sweden. Jenelius (2008) generalizes importance to consider regions in the transport system.

Sohn (2006) proposes an accessibility index that incorporates road distance and traffic volumes and uses it to assess the importance of highway links in Maryland, USA, under flood damage; the importance of a link is measured as the total decrease in this accessibility index when the link is closed. Scott et al. (2006) propose a Network Robustness Index to identify important links in highway networks, which is defined as the increase in vehicle travel time that is incurred when the link is closed. A similar measure, based on the inverse of travel cost, is defined by Qiang and Nagurney (2008). Chen et al. (2007) propose the use of a combined travel demand model incorporating trip generation, destination choice, mode choice, and route choice to assess the long-term equilibrium effects of a closure of one or more links. The consequences are calculated as the decrease of a utility-based accessibility measure. Knoop and Hoogendoorn (2007) use macroscopic simulation to study the effects of blocking links in the congested urban 
network of Rotterdam, finding that proper modeling of queue spillback is crucial for correctly identifying the most important links.

Equity issues have long been considered in various fields of transport policy, such as road and congestion pricing (e.g. Eliasson and Mattsson 2006; Langmyhr 1997), public transport provision (e.g. Hay 1993), and transportation of hazardous materials (e.g. Gopalan et al. 1990). A thorough introduction to the subject is given by Litman (2002). There are many aspects of transport equity, two of the most fundamental being horizontal and vertical equity. Horizontal equity requires that the costs or benefits being considered be distributed equally among individuals. Vertical equity requires that special consideration be given to certain disadvantaged groups of people. Factors that are often considered include age, gender, ethnic background, income, residential area, disabilities, etc. (Litman 2002).

To the author's knowledge, no study to date has fully explored the equity aspects of road network vulnerability. In their study of the Australian national transport network, Taylor et al. (2006) split the overall decrease in accessibility between different origin cities, but do not consider these inequities when ranking the links.

\section{Method}

\subsection{Scope and limitations}

The consequences of a link closure are operationalized by the increase in user travel time. Travel time serves as a crude indicator of accessibility and can easily be generalized to include changes in monetary costs and travel distance as well. All users are assumed to minimize their travel time when choosing what route to take from origin to destination. When a link is closed, travelers using that link are forced to change their travel plan. We assume that the users respond to the closure by either changing routes or, if more beneficial, by delaying their trip until the link is reopened. In reality, it is likely that some users would change travel mode or destination, or choose not to travel at all. Such a decision means that the user perceives the cost (or disutility) of such an adjustment to be lower than the cost of delaying the intended trip. Therefore, by assuming inelastic demand we obtain an upper bound on the consequences of the closure. (This may not be true in reality, since users generally do not have full information about travel conditions.) In any case, the focus of our analysis is on closures lasting a few days at most, which means that the impacts on decisions to travel or choices of mode and destination should be quite limited.

In this paper, we take a horizontal approach to equity, using the increases in user travel times to measure both the total decrease in efficiency and the inequity that is caused by a road closure. Another possibility would be to weight the travel time increases according to each user's valuation of them. This would imply that users who place a high value on travel time-a trait that is usually strongly correlated with higher income-should be given special consideration. Although the latter approach is interesting, we find the former specification more desirable for the present analysis. In any event, we lack the necessary data for the latter specification to perform the case study.

Within this setting, the only systematic features that will determine the increase in travel time of a particular user are the origin-destination pair and the desired departure time. We will 
only consider inequity in the spatial dimension, that is, inequity between users of different $O D$ pairs.

\subsection{Efficiency importance and equity importance}

The traditional way to measure link importance is to calculate the overall change of some performance measure (in our case the total increase in vehicle travel time) when the link is closed, in relation to if it would remain open. Since this captures the reduction in transport network efficiency, we will refer to this as efficiency importance, $I_{\text {eff }}$. At the most basic level we consider an origin $i$, a destination $j$ and a closure of link $k$. The total increase in travel time for all users, or equivalently vehicles, traveling between $\operatorname{OD}$ pair $(i, j)$ during the closure is denoted $\Delta T_{i j}^{k}$. The efficiency importance $I_{\text {eff }}$ of link $k$ is then defined as the total increase in vehicle travel time for all OD pairs, i.e.,

$$
I_{\mathrm{eff}}(k)=\sum_{i, j \neq 1} \Delta T_{i j}^{k}
$$

Beside the overall effect of closing the link, we are also interested in how unevenly the travel time increases are distributed among the travelers. To measure this we use the coefficient of variation, $C V$, which is a well-known inequality measure (e.g. Allison 1978). For a general population $\left\{y_{u}\right\}$, where each member $y_{u}$ has a weight $p_{u}, \sum_{u} p_{u}=1, C V$ is defined as the standard deviation $\sigma$ divided by the mean $\mu$, i.e.

$$
C V=\frac{\sigma}{\mu}=\frac{\sqrt{\sum_{u} p_{u}\left(y_{u}-\bar{y}\right)^{2}}}{\bar{y}}=\frac{\sqrt{\sum_{u} p_{u} y_{u}^{2}-\bar{y}^{2}}}{\bar{y}},
$$

where $\mu=\bar{y}=\sum_{u} p_{u} y_{u}$. Note that $C V$ is dimensionless.

Although the increase in travel time will in general depend on what time a user is departing, we will not consider the inequity between different departure times. Rather we will assume that all users of a particular OD relation receive the duration-mean increase in travel time for that OD relation. For a closure of link $k$, the mean user increase in travel time for OD relation $(i, j)$ is $\Delta T_{i j}^{k} /\left(x_{i j} \tau\right)$, where $\tau$ denotes the time from the closure occurs until the traffic situation has returned to the initial, fully functional state, and $x_{i j}$ is the average travel demand in users/vehicles per unit time during that time.

With the general notation we have one population $\left\{y_{u}^{k}\right\}$ for each link $k$, where each $u$ represents a certain OD pair, $u=(i, j)$. For a particular link $k$, the population then consists of members $y_{u}^{k}=\Delta T_{i j}^{k} /\left(x_{i j} \tau\right)$, which are weighted according to the number of users, i.e. $p_{u}=x_{i j} / x$, where $x=\sum_{i, j \neq i} x_{i j}$ denotes the total demand. The coefficient of variation 
$C V_{k}$ of this population is

$$
\begin{aligned}
C V_{k}= & \frac{\sqrt{\sum_{i, j \neq i} \frac{x_{i j}}{x}\left(\frac{\Delta T_{i j}^{k}}{x_{i j} \tau}\right)^{2}-\left(\sum_{i, j \neq i} \frac{x_{i j}}{x} \frac{\Delta T_{i j}^{k}}{x_{i j} \tau}\right)^{2}}}{\sum_{i, j \neq i} \frac{x_{i j}}{x} \frac{\Delta T_{i j}^{k}}{x_{i j} \tau}} \\
= & \sqrt{x \sum_{i, j \neq i} \frac{\left(\Delta T_{i j}^{k}\right)^{2}}{x_{i j}}-\left(\sum_{i, j \neq i} \Delta T_{i j}^{k}\right)^{2}}
\end{aligned}
$$

Our definition of equity importance, $I_{\mathrm{eq}}$, is then

$$
I_{\mathrm{eq}}(k)=C V_{k}=\frac{\sqrt{x \sum_{i, j \neq i} \frac{\left(\Delta T_{i j}^{k}\right)^{2}}{x_{i j}}-\left(\sum_{i, j \neq i} \Delta T_{i j}^{k}\right)^{2}}}{\sum_{i, j \neq i} \Delta T_{i j}^{k}} .
$$

We use the coefficient of variation, rather than (for example) the commonly used Gini coefficient, for two main reasons. First, it is possible to decompose the coefficient of variation into one within-group and one between-groups components, which drastically reduces the amount of data that must be kept in memory at the same time when performing the calculations on a computer. Second, it is relatively easy to derive properties of the measure analytically, which we will use in Section 3. The coefficient of variation and the Gini coefficient are closely related, so the impact of using one instead of the other should be small in practice (see Allison 1978, for further information).

An important property of the coefficient of variation (and most common equity measures) is that it is scale-invariant. That is, if all user travel times were multiplied by the same factor, the coefficient of variation would remain the same. The total magnitude of the consequences is thus captured solely by the efficiency importance measure, while the distribution among the users is captured solely by the equity importance measure.

\subsection{An equity-weighted importance measure}

From an equity perspective, the most important links are generally those with the smallest traffic flows, since the impacts of closing such links will be distributed over only a small number of users. Therefore, we believe, the equity importance measure $I_{\text {eq }}$ on its own is of little value in transport planning, as the total decrease in efficiency should be a major factor when determining the most important links. What is needed, then, is a form of bicriteria analysis in which the weighted efficiency importance and equity importance of a link are combined into a single 
importance measure. The method that initially springs to mind is to form a weighted sum of the two measures; however, because of the inverse dependence between efficiency importance and equity importance, such a measure will assign the highest importance to a set of links that is the union of those that cause the largest overall inefficiency and those that cause the greatest inequity, and links somewhere between the two extremes will never be important. A more suitable method, we believe, is to form a weighted product of the two measures.

In order to make the new importance measure dimensionless and the weight parameter $\alpha \in[0,1]$ easier to interpret, we first normalize the efficiency importance and equity importance measures to the interval $[0,1]$ :

$$
\begin{aligned}
& \hat{I}_{\mathrm{eff}}(k)=\frac{I_{\mathrm{eff}}(k)-\min _{l} I_{\mathrm{eff}}(l)}{\max _{l} I_{\mathrm{eff}}(l)-\min _{l} I_{\mathrm{eff}}(l)}, \\
& \hat{I}_{\mathrm{eq}}(k)=\frac{I_{\mathrm{eq}}(k)-\min _{l} I_{\mathrm{eq}}(l)}{\max _{l} I_{\mathrm{eq}}(l)-\min _{l} I_{\mathrm{eq}}(l)} .
\end{aligned}
$$

We then define the equity-weighted importance $I_{\text {ew }}$ as

$$
I_{\mathrm{ew}}(k)=\left(\hat{I}_{\mathrm{eff}}(k)\right)^{1-\alpha}\left(\hat{I}_{\mathrm{eq}}(k)\right)^{\alpha}, \quad \alpha \in[0,1] .
$$

With $\alpha=0$ the (normalized) efficiency importance is recovered, and with $\alpha=1$ the (normalized) equity importance is recovered. By adjusting $\alpha$ one can control how much weight is to be put on the equity aspect. Note also that the fact that the powers of $\hat{I}_{\text {eff }}$ and $\hat{I}_{\text {eq }}$ sum to 1 is nonrestrictive: any other combination of powers can be normalized to sum to 1 without changing the ranking of any link.

\subsection{Travel time model}

In order to calculate the importance measures, we need a model of how travel times are affected by a road closure. The model presented here is applicable to very large, mainly uncongested road networks, where computation time and memory consumption are important issues. In particular, it is assumed that the closure of a link does not affect the travel time on any other link. This approximation should be valid for most of the Swedish road network used in the case study, which is largely uncongested. In densely populated areas, however, the model is likely to underestimate the delays caused by a closure. On the other hand, the greater ability of travelers in urban areas to change mode or destination should counterbalance this effect to some extent. In any case, this should be kept in mind when considering the results of the case study presented below. For a study of the road network of Stockholm taking congestion into account, see Berdica and Mattsson (2007).

We assume that users instantly become aware of the duration of a closure. This assumption is the simplest way to avoid the other extreme where users, faced with a closure that will last only a short time, embark on very long detours instead of waiting until the link is reopened. In any case, the longer a link is closed, the less beneficial it will be to delay the trip, and the smaller the difference between the two models will be. Jenelius (2007) develops a model that takes the slow spreading of information about a closure into account, and shows that even though the 
resulting increases in travel time are larger in absolute terms than they would be if information were immediately available, the relative differences between closures of different links are virtually the same, given the same closure duration in both cases. When simply ranking the links according to their importance, the effect of the information dissemination process will thus be limited; the closure duration itself will have a much larger impact, as will be shown below.

Suppose that link $k$, located along the shortest route (assumed to be unique) between origin $i$ and destination $j$, is closed for all traffic at $t=0$ and is reopened at $t=\tau$; assume that the travel demand from $i$ to $j$ is $x_{i j}$ vehicles per unit time, constant and inelastic. During the closure, there may be either no or at least one alternative route from $i$ to $j$. If there are no alternative routes, the best a user can do is to wait until link $k$ is reopened. Henceforth, a link of this kind will be called a cut link. Since the travel demand is assumed constant over time, a user wishing to depart during the closure will, on average, be delayed $\tau / 2$ time units. The total demand during the closure is $x_{i j} \tau$ and the total increase in vehicle travel time during this period, if $k$ is a cut link, is

$$
\Delta T_{i j}^{k}=\frac{x_{i j} \tau^{2}}{2} .
$$

If the closure duration $\tau$ is longer than a day, it is likely that a large portion of the original travel demand during the later days of the closure will consist of users who have already been affected by the closure on the first day. The typical example would be commuting where the same trip is undertaken every weekday. Formula 8 above remains valid as a consequence measure for such long closures if we assume that the cost of delaying the first trip continues to increase at the same rate even after the next trip would have been made.

If there are alternative routes, a user can choose to travel along the new shortest route or to wait until link $k$ is reopened. Let $\Delta t_{i j}^{k}$ denote the difference in travel time between the new and the original shortest route, which we assume is known to the users. The delay for a user wishing to depart at some time $t \in[0, \tau]$ will be $\min \left(\Delta t_{i j}^{k}, \tau-t\right)$. If $\Delta t_{i j}^{k} \geq \tau$, all users wishing to depart during the closure will delay their trips, which gives the same result as for a cut link. If $\Delta t_{i j}^{k}<\tau$, only the users wishing to depart after $t=\tau-\Delta t_{i j}^{k}$ will benefit from delaying their trip instead of taking the detour. It is straightforward to show that the total increase in vehicle travel time in any case is

$$
\Delta T_{i j}^{k}= \begin{cases}\frac{x_{i j} \tau^{2}}{2} & \text { if } \Delta t_{i j}^{k} \geq \tau, \\ x_{i j} \Delta t_{i j}^{k}\left(\tau-\frac{\Delta t_{i j}^{k}}{2}\right) & \text { if } \Delta t_{i j}^{k}<\tau .\end{cases}
$$

It is clear from (8) and (9) that the benefit from short alternative routes will increase with the closure duration $\tau$, since the consequences will then be linear in $\tau$, while they will be quadratic in $\tau$ if there are no short alternative routes. Thus, the duration of the closure will, in general, affect the relative importance of different links.

\section{Analytical results}

Given the travel time model expressed in formulas 8 and 9, the increase in travel time for each user is restricted by the closure duration because a user can delay their trip if doing so is a better 
option than taking a detour. This is by no means a property that always holds in reality, and we shall not put too much emphasis on it in this paper. However, this bound is very prominent in the results of the case study presented below. It is therefore worthwhile to study analytically some properties of the importance measures in combination with the present travel time model-particularly the relationship between the efficiency importance and the equity importance of a link, i.e., the magnitude and the distribution of the consequences of a link closure.

Proposition 1 For every cut link $k_{c}$, there is a unique and inverse relationship between the efficiency importance $I_{\text {eff }}\left(k_{\mathrm{c}}\right)$ and the equity importance $I_{\mathrm{eq}}\left(k_{\mathrm{c}}\right)$. A proof of this proposition is presented in the Appendix.

Proposition 2 Every non-cut link $k$ will be of less or equal equity importance $I_{\text {eq }}$ than a cut link $k_{c}$ of equal efficiency importance $I_{\text {eff. }}$ A proof of this proposition is presented in the Appendix.

These results give another argument for providing redundancy around cut links: not only will the total consequences for cut links increase more rapidly with the closure duration (as shown in Section 2.4), but they will also be distributed more unevenly among the users than for other links.

\section{Case study}

To illustrate the method presented in this paper on a full scale, we have calculated the efficiency importance $I_{\text {eff }}$ and equity importance $I_{\text {eq }}$ of every link in a detailed representation of the Swedish road network. To study the impact of closure duration on both measures, we have considered two durations: a short closure of 30 minutes, and a long closure of 48 hours. Furthermore, we have calculated the equity-weighted importance $I_{\text {ew }}$ of every link for both closure durations and studied the effect of changing the weight parameter $\alpha$.

We obtained the data for the Swedish road network from the Swedish national travel demand model system SAMPERS (Beser and Algers 2001). In SAMPERS, the travel demand between different zones is calculated using nested logit choice models which have been estimated on travel surveys. The travel time of each link is calculated with user equilibrium traffic assignments in EMME/2 (using a 0.001 relative gap stop criterion), which means that initial congestion is considered in this study. The OD travel demand matrix used in our study represents the annual daily average travel demand and includes only trips made by car. Since the demand data cannot be disaggregated into different trip purposes, the matrix contains all trips and not only commuting.

The SAMPERS system divides Sweden into zones in which all trips begin and end, each zone comprising about 1000 inhabitants. For computational reasons, SAMPERS partitions the Swedish transport system into five complementary regional submodels. We have obtained a detailed representation of the entire national road transport system by merging the regional submodels while retaining all inter-regional trips. A few links in Norway and Finland have been added to provide alternative routes and reduce border effects, but we have not calculated the importance of these links. The resulting road network consists of 77769 nodes (including 8764 centroids) and 174044 directed links, and represents a very fine level of detail. For link pairs 
constituting two-way road segments, both directions are closed simultaneously, and results are presented for the link pair rather than for each direction.

The calculations were performed with specially developed software written in C++/C\#. Using a 2.0 GHz Pentium III laptop with $1 \mathrm{~GB}$ RAM, calculating the efficiency importance and equity importance of every link for a specific closure duration takes about 12 hours. These reasonable computation times could not have been obtained without the assumption that link travel times are independent of link flows.

\section{Numerical results}

The relationship between the efficiency importance $I_{\text {eff }}$ and the equity importance $I_{\text {eq }}$ was studied analytically in Section 3, where we showed that for a given $I_{\text {eff }}, I_{\text {eq }}$ is bounded from above by the cut links. This feature is prominent in Figure 1, which shows that for a 30-minute closure the shortest detour will often be longer than the closure, so the link essentially becomes a cut link. For a 48-hour closure the shortest detour is always shorter than the closure, and the upper bound becomes much less restrictive. Figure 1 also shows that $I_{\text {eq }}$ is virtually independent of the closure duration for all links, so that different closure durations merely shift the points along the $I_{\text {eff }}$ axis.
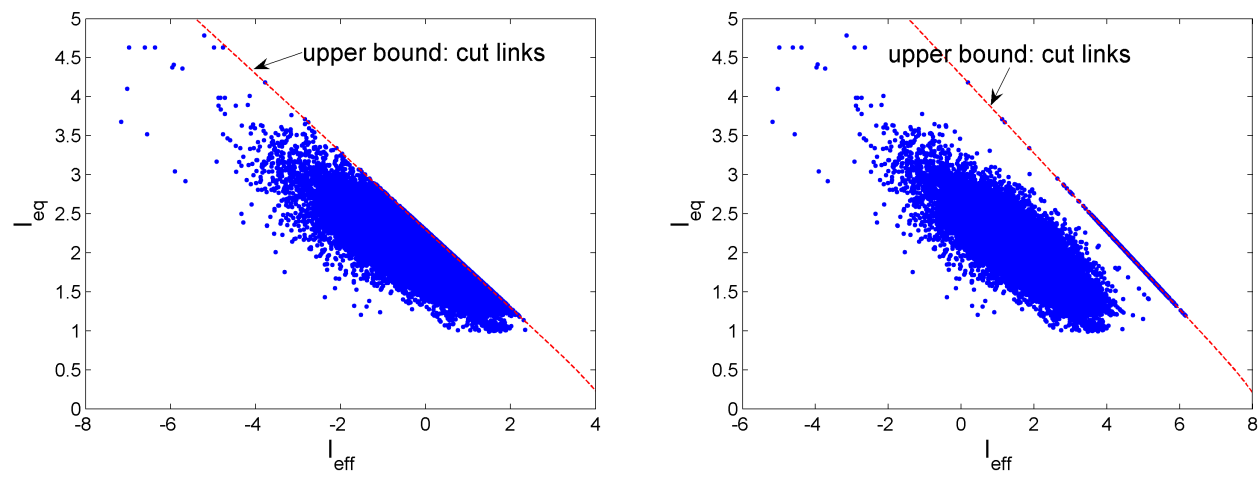

Figure 1: Efficiency importance $I_{\text {eff }}$ (vehicle hours) vs. equity importance $I_{\text {eq }}$ for every link in the Swedish road network representation. Left: Short closure $(0.5 \mathrm{~h})$. Right: Long closure $(48 \mathrm{~h})$. Logarithmic scales, axes show the order of magnitude.

Perhaps the most important feature to note, however, is the strong general inverse relationship between the two importance measures. This fundamental relationship should hold for other choices of travel time model and equity measure as well.

We now turn to the question of how the weight parameter $\alpha$ affects the importance ranking of the links according to the equity-weighted importance measure $I_{\text {ew }}$. Figure 2 shows how $I_{\text {ew }}$ depends on $\hat{I}_{\text {eff }}$ for $\alpha=0.2,0.4,0.6$ and 0.8 , respectively, for a 48-hour closure. The case $\alpha=0$ is obviously a straight line with $45^{\circ}$ inclination, and the case $\alpha=1$ is shown, for non-normalized $I_{\text {eff }}$ and $I_{\text {eq }}$, to the right in Figure 1. There is a shift between $\alpha=0.6$ and $\alpha=0.8$ where a cut link causing less inefficiency and hence more inequity than another cut link, is considered the more important link. It can be shown analytically that this shift occurs at $\alpha=2 / 3$. This value of $\alpha$ 
therefore seems to be a natural pivotal point between a regime in which efficiency is considered more important than equity and vice versa.
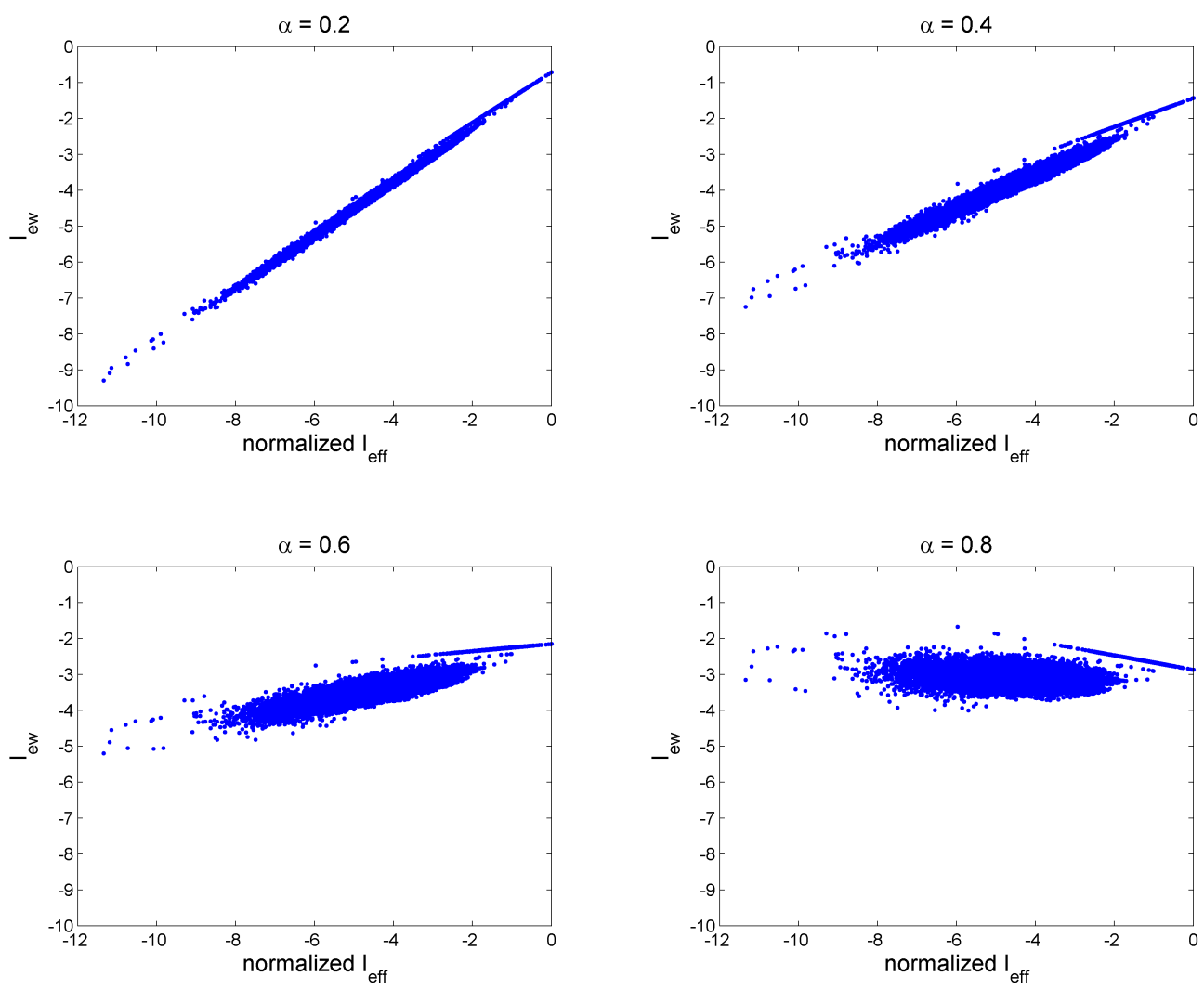

Figure 2: Normalized efficiency importance $\hat{I}_{\text {eff }}$ vs. equity-weighted importance $I_{\text {ew }}$ for every link in the Swedish road network representation and different values of the weight parameter $\alpha$. Long closure (48 h). Logarithmic scales, axes show the order of magnitude.

Figure 3 shows the pure efficiency importance of every link in the Swedish road network representation for a 48-hour closure. Sweden has three main urban regions: the Stockholm region on the east coast; the Gothenburg region on the west coast; and the Skane region in the south. As expected from the high travel demand, there are many efficiency-important links in the first two regions (the most important link of all being the Lidingö bridge in Stockholm). Skåne, however, has relatively few important links, which suggests that the road network in this region is better able to handle disruptions. Remember, however, that no congestion effects are considered in the travel time model, so many links in the urban regions should be more important in reality than what is indicated here.

Many of the main roads in Sweden are clearly visible on the map, including the E4 European highway connecting Skåne and Stockholm and continuing along the entire northeast coast, the E18 from Stockholm to Norway, the E20 connecting Stockholm-Gothenburg-Skåne, the E22 along the southeast coast, and the E45 through the northern inland region. This shows that the simple travel time model used here is sufficient to produce results that seem intuitively reasonable. There are also more isolated road links throughout the network that are important, 


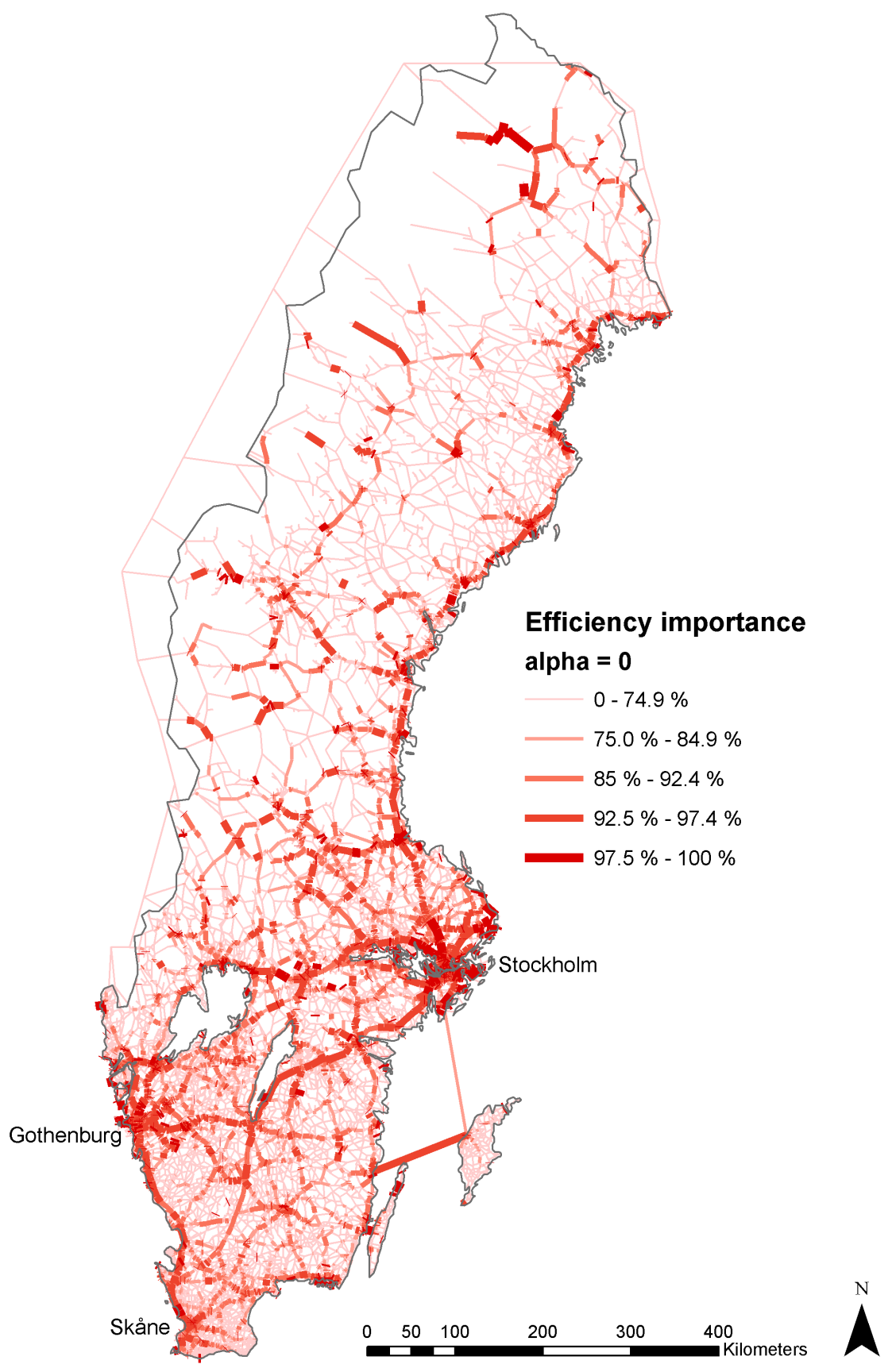

Figure 3: Efficiency importance of every link in the Swedish road network representation. Long closure ( $48 \mathrm{~h})$. The percentages indicate the percentiles of each category. 
often because they are cut links. Such results must be treated with some care, however, as they are sensitive to the network representation. In reality, there may be small roads not included in our data that could provide alternative routes; on the other hand, these would typically have very low capacities.

Figure 4 presents the equity-weighted importance $I_{\mathrm{ew}}$ where $\alpha=0.5$. The links have been divided into the same five percentile categories as in Figure 3 to facilitate comparisons (however, the equity-weighted importance measure tends to emphasize longer links, which gives quite different appearances to the maps). The value $\alpha=0.5$ was chosen arbitrarily to demonstrate the effects of incorporating equity importance. The map shows that, as with $I_{\text {eff }}$, the Stockholm and Gothenburg regions contain many important links. Some of the main roads are also still visible, although more fragmented in small sections. The most notable difference is that many links in northern Sweden, where the traffic is particularly sparse, are found to be more important. These are clearly links where users are severely affected by disruption, which causes both high inefficiency and high inequity. In southern Sweden as well, importance is transferred from the main roads to smaller local roads, which gives the map a more scattered overall appearance.

\section{Conclusion}

We have presented a method to incorporate user equity considerations when identifying the most important links in a road network. In general terms, the importance of a link is defined as the consequences for the network users when the link is closed. As a complement to measuring the total increase in vehicle travel time, denoted here as efficiency importance, we also measure the disparity in the distribution among individual users, denoted as equity importance. These two components are weighted together to form an equity-weighted importance measure.

We showed both analytically and numerically that there is a strong inverse relationship between efficiency importance and equity importance. By adjusting the weight parameter $\alpha$, the transport planner can control how much influence equity considerations should have on link importance. With the travel time model and equity measure used here, an $\alpha$ value below $2 / 3$ should be used to ensure that the overall efficiency is still the foremost concern.

The case study of the Swedish road network showed that when pure efficiency importance is considered $(\alpha=0)$, links formed by many of the main roads (in particular the European highways) are among the most important. As the value of $\alpha$ increases, importance is gradually shifted to smaller local roads with poor or no alternative routes. These results clearly tell us that if we are only concerned with the overall efficiency of the road transport system, we should focus our attention on the largest and busiest roads; if, however, we are also concerned with user equity, more attention must be given to certain local roads.

Although we did not consider congestion effects in this paper, the equity-weighted importance measure is applicable even with more refined travel time models. All that is required is to calculate the sum, standard deviation and mean of the user travel times. How the travel times are obtained, be it from measurements, micro-simulation, or (as in this case) analytical models, may of course affect the results, but not the method itself.

We mentioned in Section 1 that identifying the most important links is a natural first step in preparing a full vulnerability analysis of the road network. Further analysis would then provide estimates of the probabilities for disruptions occurring on these roads, based on the road standard, traffic load, local environment, regional weather conditions, etc. The last step, if deemed 


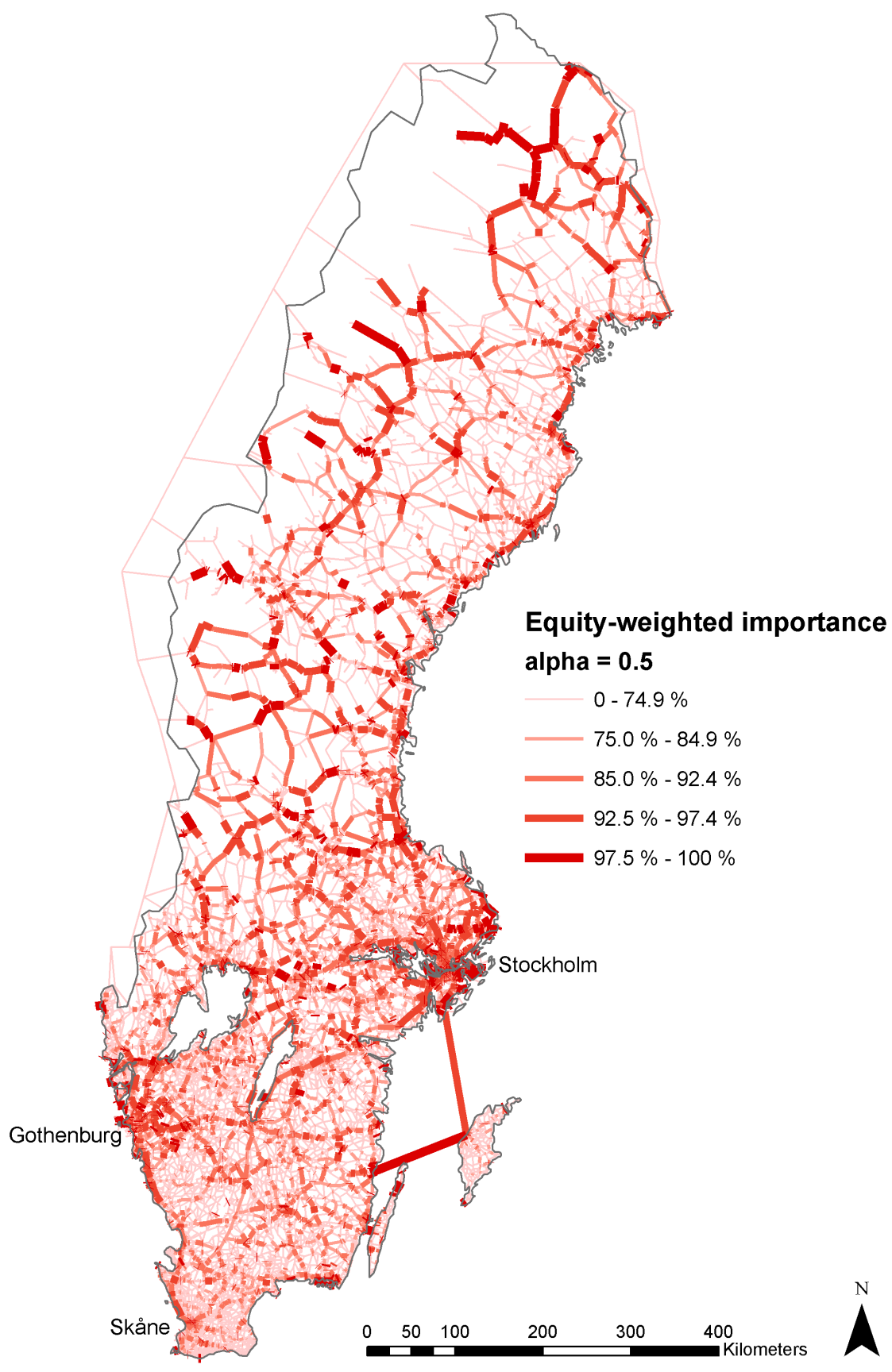

Figure 4: Equity-weighted importance of every link in the Swedish road network representation, with weight parameter $\alpha=0.5$. Long closure ( $48 \mathrm{~h}$ ). The percentages indicate the percentiles of each category. 
necessary, would be to identify the appropriate actions to be taken to reduce these vulnerabilities. Such actions would typically involve upgrading the physical road structure, enhancing traffic regulation, expanding maintenance and operational services, or building complementary roads.

If vulnerability issues are to be considered in the planning process of new road investments, they should ideally be integrated into an appraisal framework that also takes into account travel time savings, environmental impacts, traffic safety etc. For this purpose, it becomes necessary to assess the socio-economic consequences of unexpected, temporary deteriorations of the road network. Due to users' limited abilities to adapt their activity patterns, the economic impacts should be quite different from the effects of, for example, permanently closing a road, or the delays due to recurrent congestion. While multiplying increases in travel time by some appropriate value of time may be a reasonable first approach, we feel that the "value of vulnerability" is an important issue for future research.

\section{Acknowledgments}

The author would like to thank Lars-Göran Mattsson, Katja Vourenmaa Berdica, and two anonymous referees for valuable comments and suggestions. This research was financially supported by the Swedish Road Administration and the Swedish Governmental Agency for Innovation Systems (VINNOVA).

\section{References}

Allison, P. 1978. Measures of inequality. American Sociological Review, 43(6):865-880. URL http://links.jstor.org/sici?sici=0003-1224I.

Bell, M. and C. Cassir, eds. 2000. Reliability of Transport Networks. Baldock, Herts: Research Studies.

Berdica, K. 2002. An introduction to road vulnerability: What has been done, is done and should be done. Transport Policy, 9(2):117-127. doi:10.1016/S0967-070X(02)00011-2.

Berdica, K. and L.-G. Mattsson. 2007. Vulnerability: A model-based case study of the road network in stockholm. In A. Murray and T. H. Grubesic, eds., Critical Infrastructure: Reliability and Vulnerability, Advances in Spatial Science, pp. 81-106. Springer. URL http://www.springerlink.com/content/v7264h8px37350h2/.

Beser, M. and S. Algers. 2001. SAMPERS-the new Swedish national travel demand forecasting tool. In L. Lundqvist and L.-G. Mattsson, eds., National Transport Models: Recent Developments and Prospects, Advances in Spatial Science, pp. 101-118. Springer.

Chen, A., C. Yang, S. Kongsomsaksakul, and M. Lee. 2007. Network-based accessibility measures for vulnerability analysis of degradable transportation networks. Networks and Spatial Economics, 7(3):241-256. URL http://www.springerlink.com/content/ w21774970754123k/.

Eliasson, J. and L.-G. Mattsson. 2006. Equity effects of congestion pricing: Quantitative methodology and a case study for stockholm. Transportation Research Part A, 40(7):602-620. doi:10.1016/j.tra.2005.11.002. 
Gopalan, R., K. Kolluri, R. Batta, and M. Karwan. 1990. Modeling equity of risk in the transportation of hazardous materials. Operations Research, 38(6):961-973. URL http: //links.jstor.org/sici?sici=0030-364X.

Hay, A. 1993. Equity and welfare in the geography of public transport provision. Journal of Transport Geography, 1(2):95-101. doi:10.1016/0966-6923(93)90003-I.

Iida, Y. and M. Bell, eds. 2003. The Network Reliability of Transport. Oxford, UK: PergamonElsevier.

Jenelius, E. 2007. Incorporating dynamics and information in a consequence model for road network vulnerability analysis. Paper presented at the 3rd International Symposium on Transport Network Reliability (INSTR), The Hague, The Netherlands, July 19-20 2007. URL http://www.infra.kth.se/tla/projects/vulnerability/Paper_INSTR_2007.pdf.

Jenelius, E. 2008. Network structure and travel patterns: Explaining the geographical disparities of road network vulnerability. Journal of Transport Geography, 17(3):234-244. doi: 10.1016/j.jtrangeo.2008.06.002.

Jenelius, E., T. Petersen, and L.-G. Mattsson. 2006. Importance and exposure in road network vulnerability analysis. Transportation Research Part $A$, 40(7):537-560. doi:10.1016/j.tra. 2005.11.003.

Knoop, V. and S. Hoogendoorn. 2007. The need of spillback simulation in assessing robustness: Concepts and a case study with dynamic route choice. Presented at the Sixth Triennial Symposium on Transportation Analysis (Tristan), Phuket Island, Thailand.

Lam, W. 1999. Special issue on network reliability and transport modelling. Journal of Advanced Transportation, 33(2):121-251. URL http://www.advanced-transport.com/Vol33_ no2_listing.htm.

Langmyhr, T. 1997. Managing equity: The case of road pricing. Transport Policy, 4(1):25-39. doi:10.1016/S0967-070X(96)00031-5.

Litman, T. 2002. Evaluation transportation equity. Transport Policy, 8(2):50-65. URL http: //www.vtpi.org/equity.pdf.

Murray, A. and T. Grubesic, eds. 2007. Critical Infrastructure: Reliability and Vulnerability. Advances in Spatial Science. Springer. URL http://www.springerlink.com/content/r23w23/.

Qiang, Q. and A. Nagurney. 2008. A unified network performance measure with importance identification and the ranking of network components. Optimization Letters, 2(1):127-142. URL http://www.springerlink.com/content/x863m59674163435/.

Scott, D., D. Novak, L. Aultman-Hall, and F. Guo. 2006. Network robustness index: A new method for identifying critical links and evaluating the performance of transportation networks. Journal of Transport Geography, 14(3):215-227. doi:10.1016/j.jtrangeo.2005.10. 003.

Sohn, J. 2006. Evaluating the significance of highway network links under the flood damage: An accessibility approach. Transportation Research Part A, 40(6):491-506. doi:10.1016/j. tra.2005.08.006.

Sumalee, A. and F. Karauchi. 2006. Special issue on reliability and emergency issues in transportation network analysis. Networks and Spatial Economics, 6(3-4):169-357. URL http://www.springerlink.com/content/xrj811243569w088/.

Taylor, M., S. Sekhar, and G. D’Este. 2006. Application of accessibility based methods for vulnerability analysis of strategic road networks. Networks and Spatial Economics, 6(3-4):267-291. URL http://www.springerlink.com/content/02670r0004n27450/. 


\section{Appendix: Proofs of analytical results}

Proof of Proposition 1 With our travel time model, only those who initially used the cut link $k_{c}$ will suffer from delays when it is closed. The number of affected users per unit time, denoted $x_{\text {aff }}\left(k_{c}\right)$, will therefore equal the initial link flow of the closed link. The efficiency importance $I_{\text {eff }}\left(k_{c}\right)$ can be written as

$$
I_{\mathrm{eff}}\left(k_{\mathrm{c}}\right)=\sum_{i, j \neq i} \Delta T_{i j}^{k_{\mathrm{c}}}=\frac{x_{\mathrm{aff}}\left(k_{\mathrm{c}}\right) \tau^{2}}{2} .
$$

The square of the equity importance $I_{\text {eq }}\left(k_{c}\right)$ becomes

$$
I_{\mathrm{eq}}^{2}\left(k_{\mathrm{c}}\right)=\frac{x \sum_{i, j \neq i} \frac{\left(\Delta T_{i j}^{k_{\mathrm{c}}}\right)^{2}}{x_{i j}}}{\left(\sum_{i, j \neq i} \Delta T_{i j}^{k_{\mathrm{c}}}\right)^{2}}-1=\frac{x x_{\mathrm{aff}}\left(k_{\mathrm{c}}\right)\left(\tau^{2} / 2\right)^{2}}{\left(x_{\mathrm{aff}}\left(k_{\mathrm{c}}\right) \tau^{2} / 2\right)^{2}}-1=\frac{x}{x_{\mathrm{aff}}\left(k_{\mathrm{c}}\right)}-1
$$

so that

$$
I_{\text {eq }}\left(k_{\mathrm{c}}\right)=\sqrt{\frac{x}{x_{\mathrm{aff}}\left(k_{c}\right)}-1}=\sqrt{\frac{x \tau^{2} / 2}{I_{\mathrm{eff}}\left(k_{\mathrm{c}}\right)}-1},
$$

which shows the unique and inverse relationship between $I_{\text {eff }}\left(k_{c}\right)$ and $I_{\text {eq }}\left(k_{c}\right)$ for any cut link $k_{c}$.

Proof of Proposition $2 \quad I_{\text {eff }}(k)$ and $I_{\text {eq }}^{2}(k)$ of any non-cut link $k$ can be decomposed as

$$
I_{\mathrm{eff}}(k)=\sum_{(i, j) \in A_{k}} x_{i j} \tau^{2} / 2+\sum_{(i, j) \in B_{k}} x_{i j} \Delta t_{i j}^{k}\left(\tau-\Delta t_{i j}^{k} / 2\right)
$$

and

$$
I_{\mathrm{eq}}^{2}(k)=\frac{x\left(\sum_{(i, j) \in A_{k}} x_{i j}\left(\tau^{2} / 2\right)^{2}+\sum_{(i, j) \in B_{k}} x_{i j}\left(\Delta t_{i j}^{k}\left(\tau-\Delta t_{i j}^{k} / 2\right)\right)^{2}\right)}{I_{\mathrm{eff}}^{2}(k)}-1,
$$

where $A_{k}$ and $B_{k}$ are the sets of OD relations (either possibly empty) for which $\Delta t_{i j}^{k} \geq \tau$ and $0<\Delta t_{i j}^{k}<\tau$, respectively. If $\Delta t_{i j}^{k}<\tau$, then $\Delta t_{i j}^{k}\left(\tau-\Delta t_{i j}^{k} / 2\right)<\tau^{2} / 2$. Hence,

$$
\begin{aligned}
I_{\mathrm{eq}}^{2}(k) \leq & \frac{x \tau^{2} / 2\left(\sum_{(i, j) \in A_{k}} x_{i j} \tau^{2} / 2+\sum_{(i, j) \in B_{k}} x_{i j} \Delta t_{i j}^{k}\left(\tau-\Delta t_{i j}^{k} / 2\right)\right)}{I_{\mathrm{eff}}^{2}(k)}-1 \\
& =\frac{x \tau^{2} / 2}{I_{\mathrm{eff}}(k)}-1 .
\end{aligned}
$$


Since by assumption $I_{\text {eff }}(k)=I_{\text {eff }}\left(k_{c}\right),(12)$ gives

$$
I_{\mathrm{eq}}^{2}(k) \leq \frac{x \tau^{2} / 2}{I_{\mathrm{eff}}\left(k_{\mathrm{c}}\right)}-1=I_{\mathrm{eq}}^{2}\left(k_{\mathrm{c}}\right),
$$

and hence $I_{\text {eq }}(k) \leq I_{\text {eq }}\left(k_{c}\right)$, which completes the proof. 